\title{
Reference maps for anisotropy searches with AMS-02
}

\section{J. Casaus, C. Mañá and M.A. Velasco*}

Centro de Investigaciones Energéticas, Medioambientales y Tecnológicas (CIEMAT)

E-mail: jorge.casaus@ciemat.es, carlos.mana@ciemat.es,

miguelangel.velasco@ciemat.es

\begin{abstract}
The measurement of anisotropies in cosmic ray arrival directions requires knowledge of what is expected in case of an isotropic flux. This template can then be used as a reference to search for deviations from isotropy. Two types of anisotropies are commonly cited in the literature: (i) relative anisotropies, where the arrival directions of a cosmic ray sample serve as a reference and (ii) absolute anisotropies, where the reference describes the directional response of the detector to an isotropic flux. Here we discuss the advantages and shortcomings of different techniques to construct such reference maps from data. We introduce a novel method to determine the isotropic reference maps used for the measurement of absolute anisotropies with AMS-02, which can be applied to detectors with any given field of view or any particle species. The performance of this reference map will be presented in several motivated coordinate systems.
\end{abstract}

35th International Cosmic Ray Conference

10-20 July, 2017

Bexco, Busan, Korea

\footnotetext{
* Speaker.
} 


\section{Introduction}

Recent measurements of the spectra of several cosmic ray species cannot be fully explained within the current understanding of propagation mechanisms. Consequently, anisotropy studies of these species have become relevant as they provide additional information to the energy dependence of the fluxes.

Several experiments in the last decades, and more recently FERMI-LAT and PAMELA $[1,2]$ reported an increase in the positron fraction above $\sim 10 \mathrm{GeV}$. In the last years, AMS-02 experiment $[3,4]$ provided a precise measurement of the positron fraction, extending the energy range up to 500 $\mathrm{GeV}$. A plethora of models to describe the rise in the positron fraction has been proposed. For most cases, the observation requires the inclusion of primary sources, which are tipically classified into two scenarios: dark matter or astrophysical origin $[5,6,7]$. The fact that cosmic ray electrons and positrons lose their energy quickly means that they have been originated in nearby sources (within $1 \mathrm{kpc}$ ). Therefore, a detection of a dipole anisotropy may reveal primary sources of positrons and help to distinguish between an astrophysical or dark matter origin [8].

On the other hand, AMS-02 has published a precise measurement of the proton flux [9] between $1 \mathrm{GV}$ and $1.8 \mathrm{TV}$, and helium flux [10] between $1.9 \mathrm{GV}$ and 3.2 TV. The results show that both fluxes deviate from a single power law and the spectral index progressively hardens at rigidities larger than $100 \mathrm{GV}$. The magnitude of the helium spectral index is different from that of the proton, but the rigidity dependence is similar for both. In fact, proton to helium flux ratio is well described by a single power law above $45 \mathrm{GV}$. The origin of this change in the spectral index may be connected to local sources (e.g.: supernova remnants [11]) or local structures in the Galactic Magnetic Field (GMF) [12] that may induce some degree of anisotropy in the high rigidity sample. Therefore, the analysis of anisotropy for high rigidity cosmic rays may help in understanding the origin of these unexpected phenomena.

Anisotropy searches in cosmic rays provide a complementary characterisation of the new phenomena observed in their fluxes and may help to understand their origin. Typically, the dipole amplitudes expected to be measured are small (varying from $10^{-2}$ to $10^{-4}$ or lower), which means that a precise understanding of detector's behavior is needed in order not to compromise the measurement.

\section{Reference maps}

The directionality of the sample is investigated by comparing the observed distribution of arrival directions with a reference map, which represents the absence of anisotropies. Any deviation observed will be regarded as a signal. Depending on the election of the reference map, there are two types of anisotropies discussed in the literature and, in particular, applied in [13]:

- Relative anisotropies, in which a data sample is used as reference. This sample can be chosen in two different ways:

- Use another cosmic ray species as reference and study the deviations of one with respect to the other (e.g.: $\left.e^{+} / e^{-}\right)$ 
- Use same cosmic ray species but at different energies to study a possible change in the directionality with the energy (e.g.: high rigidity protons with respect to low rigidity protons)

The main advantage of relative anisotropies is that systematics of the sample and reference may totally or partially cancel. However, a signal may be totally or partially diluted if it is present in both samples.

- Absolute anisotropies, in which a true isotropic map that describes the directional response of the detector to an isotropic flux is used as reference. Computation of this true isotropic map requires a precise understanding of the detector's behavior. Absolute anisotropies provide a measurement of the intrinsic directionality of cosmic ray species.

In both cases, the flux or ratio of fluxes is described as a spherical harmonics expansion in terms of multipolar coefficients $a_{\ell m}$

$$
\Phi(\theta, \varphi)=\Phi_{0}\left(1+\sum_{\ell>0} \sum_{m=-\ell}^{m=+\ell} a_{\ell m} Y_{\ell m}(\theta, \varphi)\right)
$$

\subsection{Absolute anisotropies}

One of the main challenges in the search of anisotropies is the construction of reference maps for absolute measurements. The directional response or exposure, $\mathscr{R}(\theta, \varphi)$, of a detector [14, 15] to an isotropic flux is given by the skymap distribution of the product

$$
\mathscr{R}=\mathscr{A} \cdot \mathscr{T} \cdot \varepsilon
$$

where $\mathscr{A}$ is the effective acceptance (after applying reconstruction and selection cuts), $\mathscr{T}$ is the exposure time and $\varepsilon$ is the efficiency. Consequently, the computation of the detector's exposure requires determining the distribution of these three quantities in a certain coordinate system. In order to be sensitive to, potentially, small signals, an exhaustive knowledge of the acceptance and efficiencies is needed.

In general, the direct calculation of this amount is not easy (sometimes, even, not feasible) and indirect methods, like shuffling technique, have been proposed. Those methods attempt to create reference skymaps in a more simple manner, and, under some conditions, can substitute the true exposure map.

In particular, shuffling technique $[16,17,18]$, assumes that, under an isotropic flux, there is no correlation between the local coordinates of the particle and its arrival time to produce a simulated sample by swaping times and coordinates of detection of two real events randomly chosen. However, since real events are used, a possible anisotropy may not be completely removed in the simulated sample, leading to a decrease of sensitivity when used as reference. The capability of the technique to wash out a possible signal and, therefore, the sensitivity loss depends on the relative size between the detector's field of view and the angular scale of the anisotropy, and the operating conditions of the experiment. In the case of detectors with field of view smaller than the angular size of the anisotropies, such as AMS-02, shuffling technique cannot be used to study large scale anisotropies [19]. 


\section{Exposure maps}

\subsection{Acceptance and exposure time}

The construction of isotropic skymaps requires the knowledge of the detector's acceptance. The isotropic skymap observed by the detector is the projection, second by second, of the differential acceptance into the Galactic Coordinate System (GCS). Usually, the calculation of the differential acceptances relies on the understanding of the detector behavior through a Monte Carlo simulation. We present a technique which allows to obtain a reference map for absolute anisotropies without the computation of the acceptance.

The set of directions in local coordinates $\left(\theta_{d}, \varphi_{d}\right)$ is divided in a grid of small bins, $\Delta \Omega_{d}^{i}$, each one representing a direction in the detector frame given by the pixel's center. Each one of the acceptance bins can be regarded as a point-like detector with constant acceptance and has a corresponding isotropic skymap, $\mathscr{T}_{i}$, which can be obtained by a coordinate transformation, second by second, from the detector frame to the GCS. In the same manner, a set of skymaps in galactic coordinates for events detected in each of the acceptance bins is built.

HEALPix scheme [20] is used to divide the acceptance in equal area pixels. In our case, we use a HEALPix parameter $N_{\text {side }}=32$, which corresponds to 12288 pixels of $\sim 3 \mathrm{deg}^{2}$. In the case of AMS-02, the standard selection of electrons, positrons and protons corresponds to a cone of semi aperture of about $25 \mathrm{deg}$, which lies within 544 of these 12288 pixels. Skymaps of events and exposure in the GCS are built using the same pixelization scheme with identical HEALPix parameters. The procedure can be summarized in the following steps:

1. For each second, a coordinate transformation from local coordinates of each acceptance pixel $i$ to galactic coordinates is done: $\left(\theta_{d}, \phi_{d}\right)_{i} \rightarrow(l, b)_{i}$.

2. The direction $(l, b)_{i}$ in the skymap corresponding to the acceptance bin $i$ is filled with the value of the livetime in that second.

3. Repeat 1 and 2 for each acceptance pixel and second of the analysis.

In fact, due to geomagnetic rigidity cutoff $\left(R_{\text {cut }}\right)$, a stack of skymaps in different energy bins for each acceptance bin is built. All skymaps corresponding to energies above this $R_{\text {cut }}$ are filled with the value of livetime in that second.

The fact that AMS-02 is on board the International Space Station (ISS, which is a non-inertial reference system) guarantees that each acceptance bin has enough coverage of the galactic skymap so that an individual analysis is possible. Finally, a binned-likelihood fit combining the information of skymaps for each acceptance pixel and energy bin provides the multipolar components $a_{\ell m}$. Since acceptance is constant in each individual pixel, it can be absorbed in a normalization factor in the likelihood. As a result, the method does not require the explicit determination of the acceptance.

\subsection{Efficiency correction}

Reference maps for absolute anisotropies should include not only the projection of the acceptance into the reference system but also the understanding of the detector's behavior through changes in the efficiencies. Since the ISS passes over different positions inside Earth's magnetic 
field, the rates of cosmic rays measured by AMS-02 are very different depending on the geomagnetic cutoff. This may induce a geographical dependence of efficiencies, which may produce a spurious signal in the arrival directions of cosmic rays in GCS.

There is a correlation between the reference system of analysis and the geographical position through detector's acceptance and spacecraft orbit. Consequently, a geographical dependence of efficiencies may project into GCS and induce a spurious signal if their effect is not taken into account properly. For this reason, a precise understanding of positional dependence of efficiencies and its projection into physical systems is needed. We shall remark that, since we are looking for directionality in cosmic rays, the relevant quantity is not the average value of the efficiency but its dependence on the coordinates of analysis.

We have developed a systematic procedure to quantify and include this dependence, that, briefly consists on describing the $(\theta, \varphi)$-dependence of each efficiency with the same set of functions used to expand the flux in the analysis of anisotropies. In our case, we parametrize our efficiencies in terms of a spherical harmonic basis in the coordinate system of analysis

$$
\varepsilon(\theta, \varphi)=\varepsilon_{0}\left(1+\sum_{\ell>0} \sum_{m=-\ell}^{m=+\ell} \varepsilon_{\ell m} Y_{\ell m}(\theta, \varphi)\right)
$$

This technique has several advantages:

- Smooth description of the efficiency in a coordinate system

- Since we use the same basis as in the analysis of anisotropies, the component of the efficiency in one direction translates into the same direction in the analysis.

- No need to account for possible time dependence of the corrections, the projected effect of the effciencies along the period of analysis is obtained.

Fig. 1 and Fig. 2 show the parametrization of AMS-02 TRD ${ }^{1}$ efficiency in ISS geographical position (ISS GeoPos hereinafter) and GCS, respectively. Altough the variation of the efficiency is about $2 \%$, it has a geographical dependence with a clear decrease in regions close to geomagnetic poles, where the geomagnetic cutoff is smaller and, consequently, the rate of particles is higher. This variation projects into galactic coordinates, giving rise to spurious components if it is not taken into account.

Efficiency corrections introduce systematic uncertainties in the measurement of anisotropies, which have to be evaluated carefully (see [21] for details).

\section{Results}

\subsection{Study of the effect of efficiency corrections}

Since the variation of the efficiencies is connected to changes in environmental conditions in geographical position, their effect should be enhanced in this coordinate system, which is suitable to investigate the improvements introduced by these corrections.

\footnotetext{
${ }^{1}$ Transition Radiation Detector (TRD) is used to separate electrons and positrons from protons in AMS-02.
} 


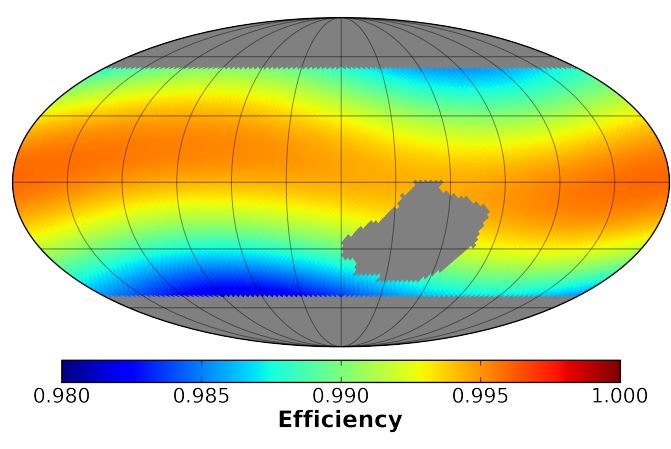

Figure 1: Parametrization of electron AMS-02 TRD efficiency up to $\ell=2$ (ISS GeoPos)

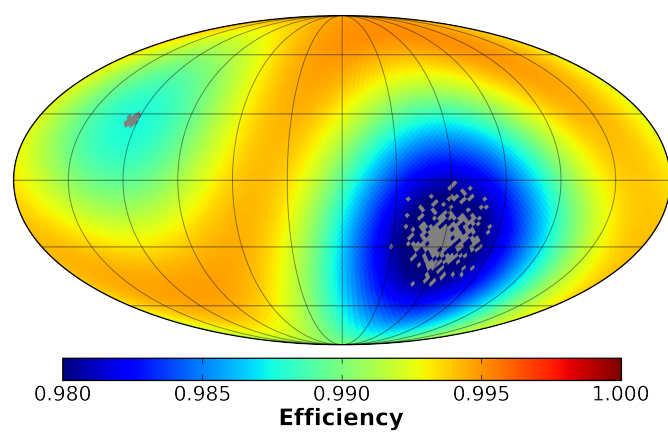

Figure 2: Parametrization of electron AMS-02 TRD efficiency up to $\ell=2$ (GCS).

Fig. 3 shows the event distribution and exposure maps for electrons selected in the energy range $16<E<350 \mathrm{GeV}$ in the ISS GeoPos. Since efficiency corrections are about few percent, exposure maps with and without corrections (Figs. $3 \mathrm{~b}$ and 3c, respectively) are, apparently, similar and, therefore, an estimator is built to quantify the differences. The effect of the corrections can be observed from the significance maps ${ }^{2}$ in Figs. 4 and 5, and significance distributions in Figs. 6 and 7. If no efficiency correction is included in the exposure map, the significance map (Fig. 4) shows a clear structure in the regions close to the geomagnetic poles, which means that the measured number of events is smaller than the expected from the exposure. The negative tail in the significance distribution (Fig. 6) corresponds to these regions. When efficiency corrections are applied, these structures are removed and, consequently, the significance distribution in Fig. 4 is now gaussian, allowing us to conclude that data is compatible with isotropy.

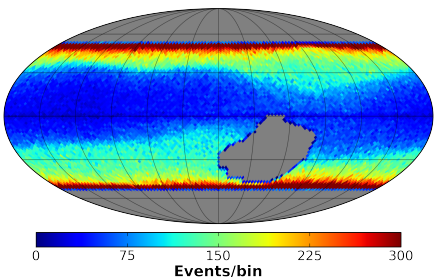

(a)

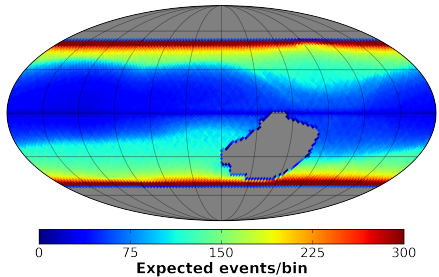

(b)

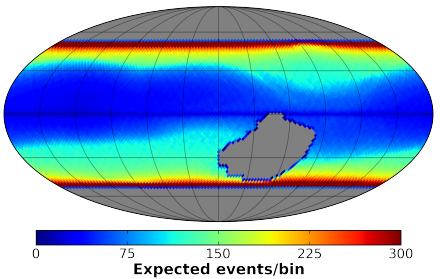

(c)

Figure 3: Event skymap (a) and exposure skymaps with (b) and without (b) for electrons selected in the energy range $16<E<350 \mathrm{GeV}$ (ISS GeoPos). Seconds in which ISS passes over the South Atlantic Anomaly (SAA) are not considered in the analysis.

\subsection{Skymaps in galactic coordinates}

Finally, the method can be applied to obtain reference maps in any physical coordinate system, such as GCS. The reference map for electrons in galactic coordinates, obtained with the procedure described above is shown in Fig. 9. From the electron skymap in Fig. 8, it is possible to obtain the significance map of the ratio, Fig. 10, which shows no evident pattern or structures.

\footnotetext{
${ }^{2}$ In order to be sensitive to possible high deviations and structures in the skymaps, significance maps are built with a smaller HEALPix parameter, $N_{\text {side }}=8$.
} 


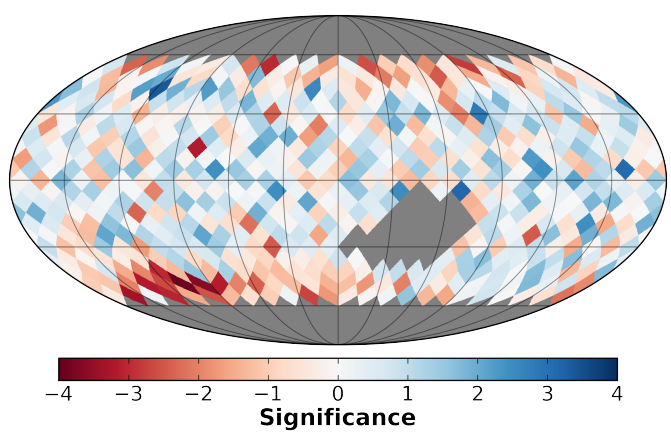

Figure 4: Significance map of electron distribution with respect to exposure map without efficiency correction (ISS GeoPos).

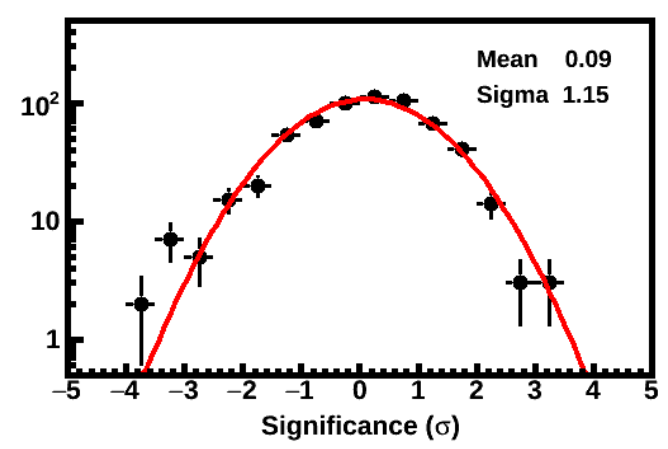

Figure 6: Significance distribution for the comparison of electrons and exposure map without correction (ISS GeoPos).

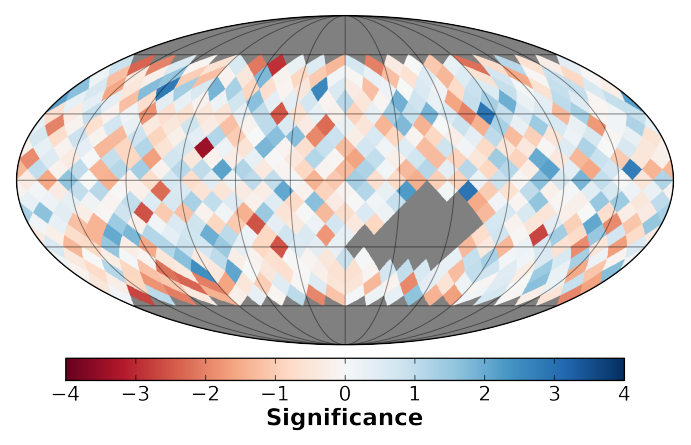

Figure 5: Significance map of electron distribution with respect to exposure map including efficiency correction (ISS GeoPos).

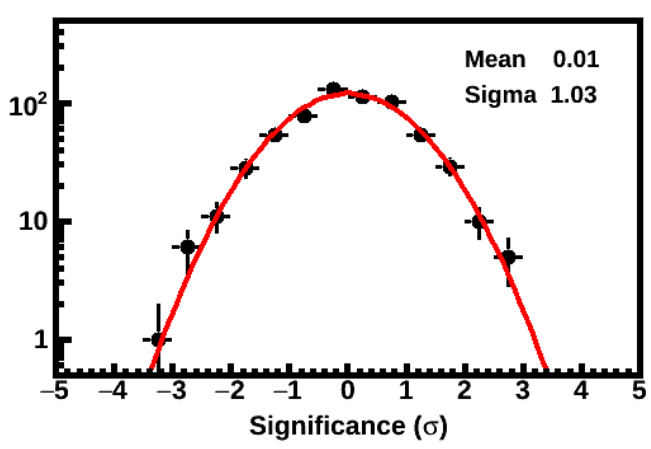

Figure 7: Significance distribution for the comparison of electrons and exposure map including corrections (ISS GeoPos).

\section{Conclusions}

One of the main challenges in the search of anisotropies is the computation of reference maps for absolute anisotropies. The determination of the directional response depends on the detector particularities and a precise understanding is needed. In some cases, indirect techniques, such as shuffling, are used, but those methods are not able to provide a valid reference map for large scale anisotropies in the case of detectors with small field of view.

The method presented in this paper, which has been applied in the case of electron, positron and proton anisotropy with AMS-02, allows to construct reference maps for absolute anisotropies regardless the detector's field of view. The keypoints of this technique are the pixelization of the acceptance in bins and the parametrization of efficiencies in terms of spherical harmonics in the same coordinate system of the analysis. This method is general and can be applied to any cosmic ray species. 


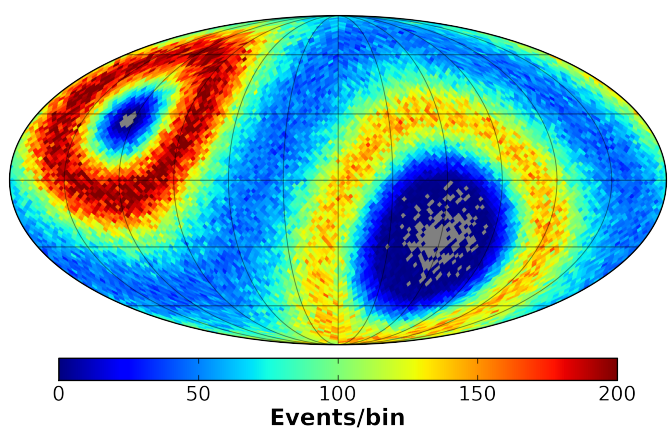

Figure 8: Skymap distribution of electrons in the energy range $16<E<350 \mathrm{GeV}$ (GCS).

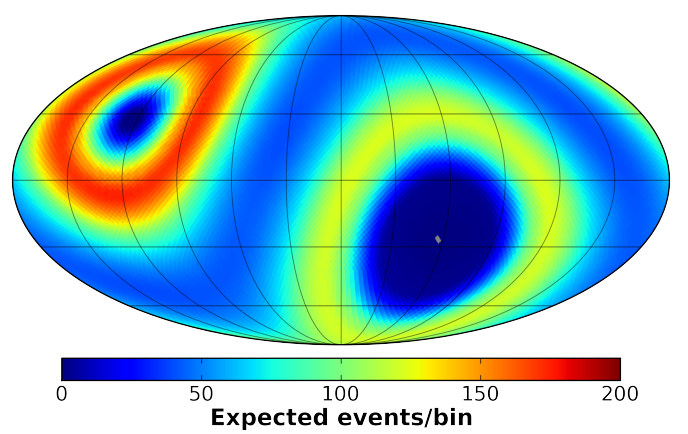

Figure 9: Reference skymap for electrons in the energy range $16<E<350 \mathrm{GeV}$ (GCS).

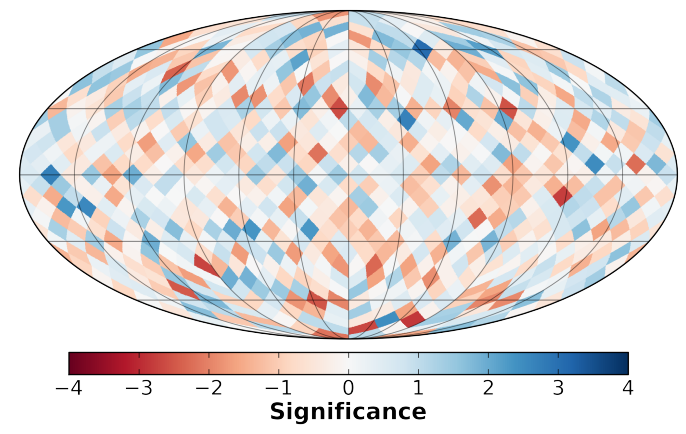

Figure 10: Significance map of electron distribution with respect to exposure map (GCS).

\section{References}

[1] O. Adriani et al. [PAMELA Collaboration], An anomalous positron abundance in cosmic rays with energies 1.5-100 GeV Nature 458, 607 (2009) [arXiv: 0810.4995 [astro-ph] ].

[2] M. Ackermann et al. [Fermi-LAT Collaboration], Measurement of Separate Cosmic-Ray Electron and Positron Spectra with the Fermi Large Area Telescope, Phys.Rev.Lett. 108, 011103 (2012) [arXiv:1109.0521 [astro-ph.HE]].

[3] M. Aguilar et al. [AMS Collaboration], First Result from the Alpha Magnetic Spectrometer on the International Space Station: Precision Measurement of the Positron Fraction in Primary Cosmic Rays of 0.5-350 GeV, Phys.Rev.Lett. 110, 141102 (2013).

[4] L. Accardo et al. [AMS Collaboration], High Statistics Measurement of the Positron Fraction in Primary Cosmic Rays of 0.5-500 GeV with the Alpha Magnetic Spectrometer on the International Space Station, Phys.Rev.Lett. 113, 121101 (2014).

[5] P. D. Serpico, Astrophysical models for the origin of the positron "excess", Astropart.Phys. 39-40, 2 (2012) [arXiv:1108.4827 [astro-ph.HE]].

[6] M. Boudaud et al., A new look at the cosmic ray positron fraction, Astronomy \& Astrophysics, $\mathbf{5 7 5}$, A67 (2015) [arXiv:1410.3799 [astro-ph.HE]]

[7] M. Di Mauro, F. Donato, N. Fornengo, A Vittino, Dark matter vs. astrophysics in the interpretation of AMS-02 electron and positron data, JCAP, 1605, 031 (2016) 
[8] I. Cernuda, Cosmic-ray electron anisotropies as a tool to discriminate between exotic and astrophysical sources, Astropart.Phys., 34, 59 (2010) [arXiv:0905.1653 [astro-ph. HE] ].

[9] M. Aguilar et al. [AMS Collaboration], Precision Measurement of the Proton Flux in Primary Cosmic Rays from Rigidity $1 \mathrm{GV}$ to $1.8 \mathrm{TV}$ with the Alpha Magnetic Spectrometer on the International Space Station, Phys.Rev.Lett. 114, 171103 (2015).

[10] M. Aguilar et al. [AMS Collaboration], Precision Measurement of the Helium Flux in Primary Cosmic Rays of Rigidities 1.9 GV to 3 TV with the Alpha Magnetic Spectrometer on the International Space Station, Phys.Rev.Lett. 115, 211101 (2015).

[11] P. Blasi, E. Amato, Diffusive propagation of cosmic rays from supernova remnants in the Galaxy. II: anisotropy, JCAP 2012, 01 (2012) [arXiv:1105.4529 [astro-ph. HE]].

[12] E. Battaner, J. Castellano, M. Masip, Galactic Magnetic Fields and the Large-Scale Anisotropy at Milagro, Astrophys.J. 703, 1 L90 (2009) [arXiv:0907.2889 [astro-ph.HE]].

[13] I. Gebauer, Measurement of anisotropies in cosmic ray arrival directions with the Alpha Magnetic Spectrometer on the ISS, this volume.

[14] J.D. Sullivan et al., Geometric factor and directional response of single and multi-element particle telescopes, Nucl. Instrum. Meth., 95, 5 (1971).

[15] D.E. Alexandreas et al., Point source search techniques in ultra high energy gamma ray astronomy, Nucl. Instrum. Meth. A, 328, 570 (1993).

[16] M. Ackermann et al. [Fermi-LAT Collaboration], Searches for Cosmic-Ray Electron Anisotropies with the Fermi Large Area Telescope, Phys.Rev., D 82, 092003 (2010) [arXiv: 1008.5119 [astro-ph. HE]].

[17] R.W. Clay, B.R. Dawson, G.J. Thornton, Directional reconstruction and anisotropy studies, Comptes Rendus Physique 5, 473 (2004)

[18] O. Deligny, F. Salamida, Searches for large-scale anisotropies of cosmic rays: Harmonic analysis and shuffling technique, Astropart.Phys., 46, 40 (2013) [arXiv:1305.5401 [astro-ph. IM] ].

[19] M.A. Velasco, PhD thesis, Universidad Complutense de Madrid, in preparation.

[20] K. M. Górski et al., HEALPix: A Framework for High-Resolution Discretization and Fast Analysis of Data Distributed on the Sphere, Astrophys.J. 622, 759 (2005) [arXiv: astro-ph/ 0409513 [astro-ph]].

[21] K.F. Bindel, I. Gebauer, M. Graziani, S. Zeissler, Study of systematics in anisotropy searches with $A M S-02$, this volume. 